\title{
A Fault Diagnosis Model of Surface to Air Missile Equipment Based on Wavelet Transformation and Support Vector Machine
}

\author{
ZHHENG Ni ${ }^{1}$, ZHANG Lin ${ }^{1}$, ZHANG Bo ${ }^{1}$, WANG Wenfeng ${ }^{1, a}$, SHI Wenjie ${ }^{1}, \mathrm{Si} \mathrm{Wei}^{2}$ \\ ${ }^{1}$ Air Defense and Antimissile Institute, Air Force Engineering University, Xi'an710051, China \\ 2. The Research Institute on General Development and Evaluation of Equipment, EAAF of PLA, Beijing 100191, \\ China
}

\begin{abstract}
At present, the fault signals of surface to air missile equipment are hard to collect and the accuracy of fault diagnosis is very low. To solve the above problems, based on the superiority of wavelet transformation on processing non-stationary signals and the advantage of SVM on pattern classification, this paper proposes a fault diagnosis model and takes the typical analog circuit diagnosis of one power distribution system as an example to verify the fault diagnosis model based on Wavelet Transformation and SVM. The simulation results show that the model is able to achieve fault diagnosis based on a small amount of training samples, which improves the accuracy of fault diagnosis.
\end{abstract}

\section{Introduction}

The surface to air missile equipment is a kind of complex equipment with mechanical, electronic and hydraulic system. The operational effectiveness of the equipment will be affected or even disabled when any part of the equipment breaks down. The combat readiness intact rate of the equipment can be improved by applying condition-based maintenance to avoid burst faults [1]. Because the early fault signal of surface to air missile equipment is feeble which can be easily affected by noise and detection error, the accurate fault state of the equipment is unable to be acquired without special processing. Among many methods of fault diagnosis, Wavelet Transformation theory and SVM are widely used and both achieve good effect. The Wavelet Transformation is an important means for non-stationary signal processing which can easily separate the monitory point's original signal and noise background to achieve the purpose of signal denoising [2]. Taking the advantage of generalization performance of SVM, the low dimensional inseparable problem can be mapped to high-dimensional space to realize the separability through kernel function skills, which better solves the problems such as nonlinearity, small samples and local minimum points [3-4]. In this paper, a fault diagnosis model based on wavelet transformation and SVM is proposed by taking the power distribution system of surface to air missile equipment as the object of study. This model uses wavelet transformation to denoise fault signals and uses SVM to classify fault feature vector, which provides an effective method for fault diagnosis of surface to air missile equipment and lays the foundation of condition-based maintenance and PHM.

\footnotetext{
${ }^{\mathrm{a} C}$ Corresponding author : dreamland_0628@163.com
} 


\section{Design of Fault Diagnosis Model}

\subsection{The Basicprinciple of Wavelet Transformation}

Wavelet Transformation is a local transformation on time and dimension, which can analyze signal from multi-dimension by flexing and translation to assign low-frequency signals lower time resolution and higher frequency resolution, in the meantime, to assign high-frequency signal higher time resolution and lower frequency resolution. The threshold-denoising method based on Wavelet Transformation is a common and effective method. The effective signal can be reconstructed by utilizing the huge difference feature between effective signal and noise signal, conversing noise signal by wavelet basis function, processing all resolved wavelet parameters by threshold and reverse-conversation.

In [5], the definition of continuous wavelet transformation and reverse-transformation is introduced in details. The continuous wavelet transformation is

$$
\left(W_{\psi} f\right)(a, b)=\left\langle f, \psi_{a, b}\right\rangle=|a|^{-1 / 2} \int_{R} f(t) \psi^{*}\left(\frac{t-a}{b}\right) d t
$$

The wavelet reverse-transformation is

$$
f(t)=\frac{1}{C_{\psi}} \int_{-\infty}^{+\infty} \int_{-\infty}^{+\infty}\left(W_{\psi} f\right)(a, b) \psi_{a, b}(t) \frac{d a}{a^{2}} d b
$$

The continuous wavelet transformation is mainly applied to the oretical study and analysis. The discrete format is generally used in numerical calculation. The discrete wavelet transformation is

$$
\left(W_{\psi} f\right)(a, b)=\left\langle f, \psi_{a, b}\right\rangle=\left|a_{0}\right|^{-m / 2} \int_{R} f(t) \bar{\psi}\left(a_{0}^{-m} t-n b_{0}\right) d t
$$

The discrete wavelet re-transformation is

$$
f(t)=c \sum_{-\infty}^{+\infty} \sum_{-\infty}^{+\infty}\left(W_{\psi} f\right)(a, b) \psi_{m, n}(t)
$$

\subsection{The Basic Principle of SVM}

The main idea of SVM is to find an optimal hyper plane to separate different samples correctly [6]. SVM can be divided into linear separability and non-linear inseparability. Generally, actual operating system such as power distribution system belongs to the nonlinear inseparable problem. In order to realize SVM under the circumstance of non-linear inseparability, a non-linear transformation is needed to map the feature sample which has already been wavelet denoised and re-transformed to high feature space. Then the linear SVM is applied to fault classification.

According to the basic principle of SVM, the optimal linear classification function is needed to realize the fault classification and to achieve the purpose of diagnosis [7].

$$
f(x)=\operatorname{sign}[(w \cdot \theta(x)+b)]
$$

In the formula, wis the weight vector and $b$ is the deviation or classification threshold.

To meet the structural risk minimization, $w$ and $b$ need to be found to make the formula (6)hold.

$$
\min R=\frac{1}{2} w^{T} w+C \cdot R_{P}
$$

where, $w^{T} w$ is the complexity of the control model. $C$ is the penalty factor. $R_{p}$ is the error control function. The loss function which is chosen in the optimization goal is the 2 -norm of error $\zeta$, then the fault diagnosis model can be optimized as 


$$
\begin{aligned}
& \min Q(w, b, \xi)=\frac{1}{2} w^{T} w+\frac{1}{2} C \cdot \sum_{i=1}^{N} \xi_{i}^{2} \\
& \text { st. } \quad y_{i}\left[w^{T} \cdot \theta\left(x_{i}\right)+b\right]=1-\xi, i=1,2, \ldots, N
\end{aligned}
$$

Due to the condition that $w$ may be infinite dimensional, it is very difficult to solve the above formula. Thus, formula(7) can be converted to following equations.

$$
\left[\begin{array}{cc}
0 & \boldsymbol{I}^{\mathrm{T}} \\
\boldsymbol{I} & K+C^{-1} I
\end{array}\right]\left[\begin{array}{l}
\boldsymbol{b} \\
\boldsymbol{a}
\end{array}\right]=\left[\begin{array}{l}
0 \\
\boldsymbol{Y}
\end{array}\right]
$$

where, $\boldsymbol{I}=[1,1, \ldots, 1]^{T}, \boldsymbol{Y}=\left[y_{1}, y_{2}, \ldots, y_{N}\right]^{T}, \boldsymbol{a}=\left[a_{1}, a_{2}, \ldots, a_{N}\right]^{T}, K=\theta\left(x_{i}\right)^{T} \theta\left(x_{j}\right)$ is the Kernel function.

Calculation difficulty will be increased when takes the polynomial kernel function at a higher order [8-9]. The precision of radial basis kernel function is higher than sigmoid kernel function, so the radial basis kernel function $K\left(x, x^{\prime}\right)=\exp \left(-\gamma\left\|x-x^{\prime}\right\|^{2}\right)(\gamma>0)$ is chosen. $\gamma$ is the coefficient of control radius. The least square method is used to get the value of $a$ and $b$, then the fault classification function can be got after wavelet denoising.

$$
f(x)=\operatorname{sgn}\left[\sum_{i=1}^{N} a_{i} K\left(x, x_{i}\right)+b\right]
$$

\subsection{The Design of Fault Diagnosis Model based on Wavelet Transformation and SVM}

The fault diagnosis schematic based on Wavelet Transformation and SVM is shown in Figure 1. Firstly the Wavelet Transformation is used to denoise the extractive fault signal and to reconstruct the signal. The fault information is extracted to build fault feature vector set. The SVM classifier is constructed by using the method of one to one. At last, the sample data is inputted into the fault diagnosis model to forecast and analyze to output the diagnosis result.

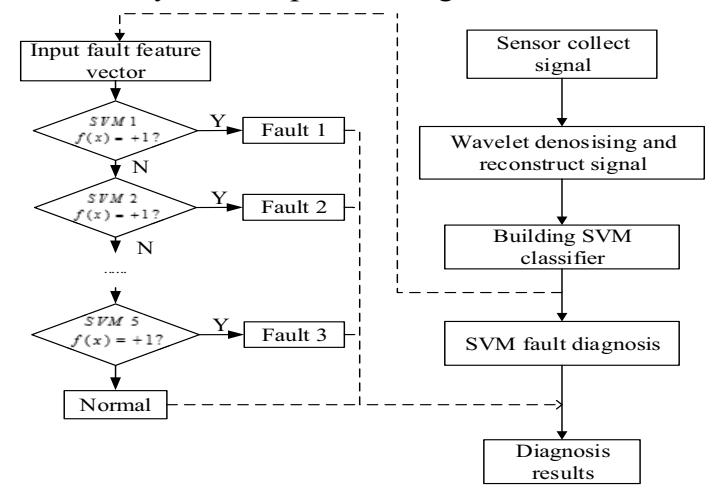

Figure 1. The schematic diagram of fault diagnosis model based on Wavelet Transformation and SVM.

\section{Simulation Analysis}

According to the dimension of circuit fault feature vector and the circuit fault mode, combining the expert experience and the characteristics of the equipment, the method of fault diagnosis based on the bottom node monitoring model is selected. After the extraction of the test node's signal, denoising technique will be applied to construct the fault diagnosis model based on SVM, so as to achieve fault diagnosis.

Taking the typical analog circuit diagnosis of one power distribution system as an example to verify the fault diagnosis model based on Wavelet Transformation and SVM, as shown in Figure2. The circuit is composed of four filters and an adder, which is divided into five parts $H_{1}, H_{2}, L_{1}, A_{1}$ and 
$L_{2}$ respectively. Monitoring nodes are $N_{1}, N_{2}, N_{3}, N_{4}$ and $N_{5}$. Fault types are fault $H_{1}$, fault $H_{2}$, fault $L_{1}$, fault $A_{1}$ and fault $L_{2}$. According to the characteristics of the hypothesis circuit, the sample data can be trained by 5 degrees SVM fault classifier to judge the circuit state.

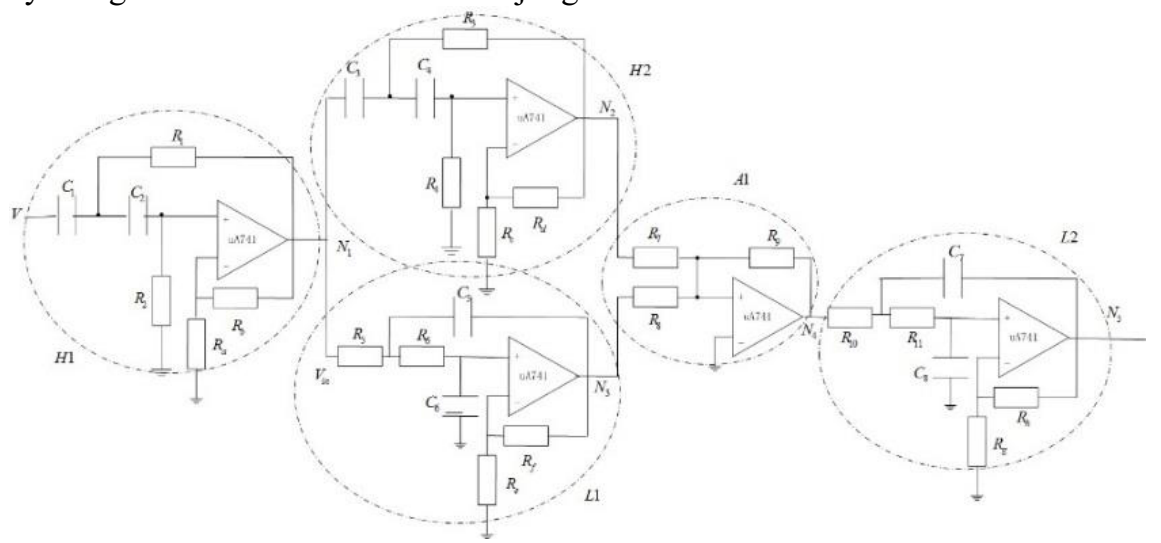

Figure 2. The typical analog circuit of one surface to air missile power distribution system.

\subsection{Parameter Selection}

\subsubsection{The performance compare of wavelet denoising}

Taking $N_{1}$ as the monitor node and the voltage signal as the feature vector, different denoising methods are analyzed separately based on the same voltage signal and the results are shown in Figure 3. It indicates that the soft threshold method is better than the other two methods. Hence, soft threshold method is chosen in the paper. The wavelet basis has a determining influence on the effect of wavelet transformation. Test the Synm N wavelet class and the DbN wavelet class based on the same test signal [10].Comparing the results, the former one is proved to be better than the latter one. Simulating and analyzing the Sym wavelet basis and the Db wavelet basis, the results are shown in Figure 4.
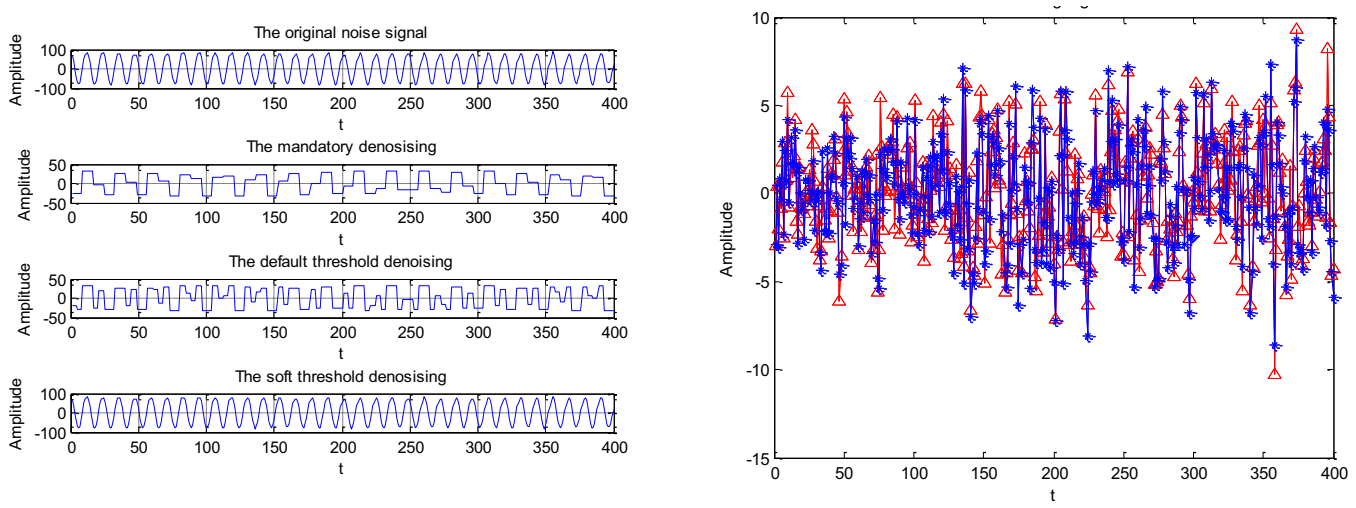

Figure 3.Denoising effect figures of three modes.

Figure 4.Denosising curve.

Using Sym wavelet basis to denoise:

$$
S N R_{\text {sym }}=18.5476(\mathrm{~dB}), \mathrm{A}_{\mathrm{sym}}=1.0842, \mathrm{MSE}_{\mathrm{sym}}=6.3896
$$

Using $\mathrm{Db}$ wavelet basis to denoise:

$$
S N R_{d b}=17.6567(\mathrm{~dB}), \mathrm{A}_{\mathrm{db}}=1.0584, \mathrm{MSE}_{\mathrm{db}}=6.9897
$$

By comparison and analysis, the effect of Sym is slightly better. Hence, Sym wavelet basis is selected in the paper. 


\subsubsection{The selection of parameter $\gamma$ in SVM}

The value of the constant $C$ which was pre-determined in radial basis function has no influence on the result, while the value of the parameter $\gamma$ does have a great one. In this paper Grid-Search is applied to the optimization of parameter $\gamma$ in SVM kernel function. The specific steps are:

Step1: When $C=10$, according to the SVM principle, the maximum range of the parameter $\gamma$ is $\gamma \in[0: 0.1: 1]$.

Step2:Create $1 * 100$ network planes and 100 parameter pairs $\left(C_{i}, \gamma_{i}\right)$ within this range, use all node parameters as learning samples and acquire learning errors after putting them in SVM, the pair $\left(C_{i}\right.$, $\left.\gamma_{i}\right)_{\min }$ corresponds to the node which has the minimum error will be adopted.

Step3: When the learning accuracy cannot meet the requirements, regard $\left(C_{i}, \gamma_{i}\right)_{\min }$ as a center, and wide the grid width with $0.01 \sim 5$ times to reconstruct parameter pairs $\left(C_{i}, \gamma_{i}\right)$. Finally the required accuracy will be achieved via optimizing the parameter $\gamma$ in SVM.

\subsection{Fault Simulation}

Taking node $N_{1}$ for example, the voltage signal is extracted by the sensor acquisition. The signal is shown in Figure 5 after applying the Sym wavelet basis and the soft threshold method to denoise.
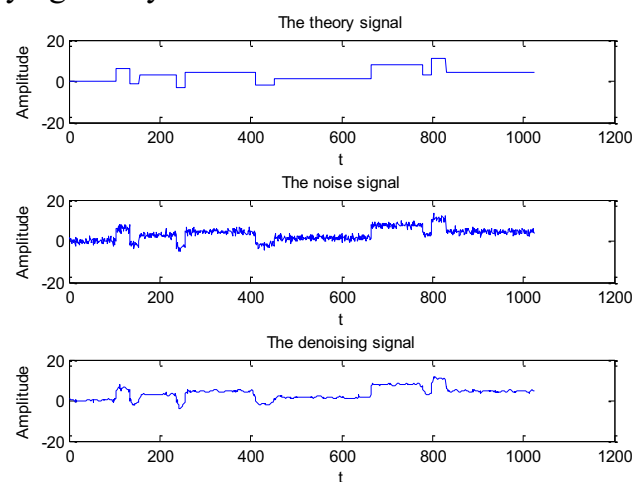

Figure 5. The collection and denosising of node $N_{1}$.

\subsection{Results analysis}

Apply the fault diagnosis model and the parameter was confirmed by using Grid-Search optimization method. The results of training and testing are shown in Table 1.

Table.1 The Results of Training and Testing.

\begin{tabular}{|c|c|c|c|c|c|c|}
\hline \multirow{2}{*}{ SVM } & $\begin{array}{c}\text { Circuit } \\
\text { state }\end{array}$ & $\begin{array}{c}\text { Testing } \\
\text { samples }\end{array}$ & $\begin{array}{c}\text { Correct } \\
\text { numbers }\end{array}$ & $\begin{array}{c}\text { Accuracy } \\
\mathbf{\%}\end{array}$ & $\begin{array}{c}\text { Operation time } \\
(\mathbf{s})\end{array}$ & $\begin{array}{c}\text { Optimal parameter } \\
\left(C_{i}, \gamma_{j}\right)\end{array}$ \\
\hline \multirow{2}{*}{$\mathrm{SVM}_{1}$} & Normal & 25 & 22 & 88 & 23.5 & $(56.172,0.1854)$ \\
\cline { 2 - 7 } & Fault & 13 & 13 & 100 & & \\
\hline \multirow{2}{*}{$\mathrm{SVM}_{2}$} & Normal & 15 & 14 & 93.3 & 25.3 & $(38.543,0.6787)$ \\
\cline { 2 - 7 } & Fault & 25 & 22 & 88 & & \\
\hline \multirow{2}{*}{$\mathrm{SVM}_{3}$} & Normal & 10 & 8 & 80 & 22.8 & $(251.3,1.0583)$ \\
\cline { 2 - 7 } & Fault & 30 & 28 & 93.3 & & $(267.4,0.1864)$ \\
\hline \multirow{2}{*}{$\mathrm{SVM}_{4}$} & Normal & 10 & 9 & 90 & 25.3 & \\
\cline { 2 - 7 } & Fault & 30 & 28 & 93.3 & & \\
\hline \multirow{2}{*}{$\mathrm{SVM}_{5}$} & Normal & 15 & 13 & 86.7 & 24.7 & \\
\cline { 2 - 7 } & Fault & 25 & 23 & 92 & & \\
\hline
\end{tabular}


Monte-Carloanalysis was carried out on the circuit and the sampling time was $10 \mathrm{~s}$. Each kind of fault classification is analysed for 120 times and the number of fault response data samples was total 600. Two-thirds samples were used for training and one third for testing.After the training of two-thirds sample data, the accuracy rate of the circuit's SVM fault diagnosis model is about $90 \%$, which can meet the requirement of fault diagnosis for power distribution system.

\section{Conclusions}

To solve the problem of fault diagnosis of the surface to air missile equipment, taking the power distribution system as the study object, a fault diagnosis model based on Wavelet Transformation and SVM is established. Different wavelet basises and denoising method are compared. According to the characteristics of the power distribution system, the parameter of SVM's kernel function is optimized and five kinds of fault feature vector sets are trained based on the feature vectors from the monitoring node $N_{1}$. Simulations are made on the typical analog circuit of one surface to air missile power distribution system. Results show that the accuracy of the fault diagnosis model based on Wavelet Transformation and SVM is about $90 \%$, which shows a good prospect in the fault diagnosis of the surface to air missile equipment.

\section{References}

1. ZHANG Lin,SUN Anquan,WANG Tianyi,et al. Intelligent fault diagnosis of certain missile equipment. Journal of Central South University(Science and Technology), 216-220.(2013)

2. LI Xue-liang,LIU Shi-bin,CHENdong, QIU Song-song. Denoising of LAPS signal based on wavelet transform. Journal of Optoelectronics • Laser,25(5):835-839. (2014)

3. GU Wen-cheng;CHAIBao-ren;TENG Yan-ping. Research on Support Vector Machine Based on Particle Swarm Optimization.Transactions of Beijing Institute of Technology, 34(7):705-709.(2014)

4. Lin Zhang, Liu Tao, etc. Research on Fault Diagnosis of Weapon Equipment Based on SVM.2012International Conference on Intelligent System and Applied Material GSAM,1242-1245. (2012)

5. ZENG Zuo-qin. Application of Wavelet Transform for Data Processing in Making and Breaking Test.Electrotechnics Electric.2:42-47.(2012)

6. $\mathrm{Hu}$ Liang-mou. Support Vector Machine fault diagnosis and control technology.Beijing: Academic Press.(2011)

7. An-quan Sun, Lin Zhang, Wen-feng Wang, et al. Robust FDI for a Class of Nonlinear Networked Systems with ROQs. Mathematical Problems in Engineering, 1-8.(2014)

8. WANG Chun-lin,ZHOUhao,LIGuo-neng.etc. Combining Support Vector Machine and Genetic Algorithm to Predict Ash Fusion Temperature.Proceedings of the CSEE, 08:11-15.(2007)

9. Lin H T, Lin C J. A study on sigmoid kernels forSVM and the training of non-PSD kernels by SMO-type methods[EB]. http:/www.csie.ntu. edu.Tw/ cilin/papers.Html.(2003)

10. ZHENG Jun,HOU Rui-feng. Selection of Wavelet Base in Denoising of Wavelet Transform. Journal of Shenyang University,21(2):108-110.(2009) 